\title{
Rapid survey of nuclear quadrupole resonance by broadband excitation with comb modulation and dual-mode acquisition
}

\author{
Yuta Hibe $^{1}$, Yasuto Noda ${ }^{1}$, K. Takegoshi ${ }^{1}$, and Kazuyuki Takeda ${ }^{1}$ \\ ${ }^{1}$ Division of Chemistry, Graduate School of Science, Kyoto University, \\ 606-8502 Kyoto, Japan
}

\begin{abstract}
Nuclear Quadrupole Resonance (NQR) provides spectra carrying information as to the electric-field gradient around nuclei with a spin quantum number $I>1 / 2$ and offers helpful clues toward characterizing the electronic structure of materials of chemical interest. A major challenge in NQR is finding hitherto unknown resonance frequencies, which can scatter over a wide range, requiring time consuming repetitive measurements with stepwise frequency increments. Here, we report on an efficient, two-step NQR protocol by bringing rapid-scan and frequency-comb together. In the first step, wideband excitation and simultaneous signal acquisition, both realized by a non-adiabatic, frequency-swept hyperbolic secant (HS) pulse with comb modulation, offers a clue for the existence/absence of the resonance within the frequency region under investigation. When and only when the sign of the resonance has been detected, the second step is implemented to compensate the limited detection bandwidth of the first and to unambiguously determine the NQR frequency. We also study the spin dynamics under the comb-modulated HS pulse by numerical simulations, and experimentally demonstrate the feasibility of the proposed scheme, which is referred to as RApid-Scan with GApped excitation with Dual-mode Operation (RASGADO) NQR.
\end{abstract}


Atomic nuclei with a spin quantum number $I>1 / 2$ possess a quadrupole moment, and a quadrupolar interaction arises in the presence of electric-field gradient (efg), causing quadrupolar energy splitting. Nuclear Quadrupole Resonance (NQR) is a branch of magnetic resonance spectroscopy, which allows one to access the quadrupolar splitting in the absence of the externally applied magnetic field. Since the experimentally accessible quadrupolar parameters are determined by the efg tensor and thereby by the electronic structure around the nuclei under study, NQR provides a useful tool for characterizing materials of chemical interest.

However, the very process of finding hitherto unknown resonance conditions can be painstaking, due to that the resonance frequencies of a given quadrupolar isotope can scatter over a wide range. The necessity of the frequency-stepping method by implementing a number of separate measurements for various stepwise carrier frequencies led to development of a sophisticated Interleaved Subspectrum Sampling (ISS) by Scharfetter et al $[1,2]$. In ISS, excitation at a certain frequency is followed by excitation at another, far-different frequency to eventually cover the full spectral range of interest. Since the time interval one needs to wait for the recovery of the saturated magnetization by the previous pulse excitation is considerably reduced, one can save the overall measurement time.

In this work, we report on another labor-saving strategy for the search of NQR signals. The idea of the new scheme is to perform a quick check of the existence of resonance by a comb-modulated hyperbolic secant (HS) pulse serving for broadband rapid-scan[3] excitation, and when and only when the sign of the resonance has been recognized, the experimental sequence is switched to another mode that allows unambiguous determination of the NQR frequency. This method, which may be called as RApid-Scan with GApped excitation with Dual-mode Operation (RASGADO) NQR, is based on that NQR lines in general appear only sparsely within the entire spectral range. For brevity, we call the first and the second parts of the two-step operation of RASGADO NQR as the coarse survey and the fine survey modes, respectively. Separation of the experiment into these modes significantly reduces the net experimental time when, as is usually the case, the NQR lines appear only in a restricted region of the full spectral range under study. The advantage of the RASGADO scheme comes from the broad excitation bandwidth in the coarse survey 
realized by comb-modulation, and from the information that can be obtained during the coarse survey regarding the existence/absence of the resonance. This contrasts with the conventional frequency stepping approaches, in which the experimental effort is devoted equally to every region of the full spectral range.

Comb modulation, which is widely used in laser optics and has recently been applied to rf pulses in NMR as well[4,5], provides a periodic, broadband excitation profile in the frequency domain. As we show below, the comb-modulated HS pulse exhibits a wideband excitation profile with periodically replicated Fourier-transformed HS functions. In NMR and NQR, we can also take advantage of the prominent feature, namely, the gap, of comb excitation during which the rf transmitter is switched off. We show that such gaps could be fully exploited for windows of data sampling.

The comb-modulated HS pulse is reminiscent of gapped HS excitation in magnetic resonance imaging (MRI) by sweep imaging with Fourier transformation (SWIFT), extensively explored by Idiyatullin et al[6]. In SWIFT MRI, the bandwidth of interest is within the spectral width given by the inverse of the interval between the gaps, and those frequency components lying outside the spectral range are just discarded. Conversely, in RASGADO NQR, we assertively make use of all Fourier components of the excitation pulse spreading over the wide range introduced by the comb modulation.

\section{Principle}

\subsection{Comb-modulated HS pulse}

Let us consider a quadrupolar nucleus and suppose that its NQR frequency $\omega_{0}$ is unknown, but for simplicity, that the resonance is known to be somewhere within the bandwidth $\Delta \Omega$ of the tank circuit of the probe. Our goal is to fully exploit an HS pulse [7, 8] with comb modulation for broadband excitation and simultaneous signal acquisition. An HS pulse $g_{1}(t)$ is expressed in the rotating frame with respect to its center frequency by a complex function as $[7,8]$

$$
g_{1}(t)=\omega_{\max }[\operatorname{sech}(\beta t)]^{1-i \mu},
$$


(a)

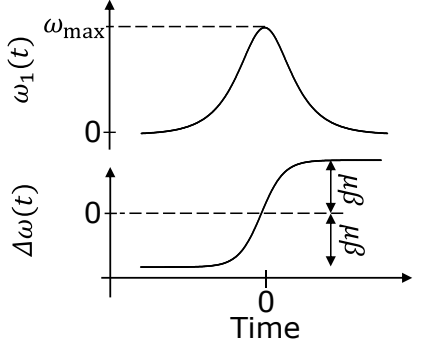

(b)

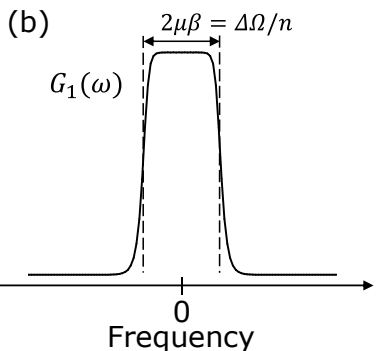

Figure 1: (a) Profiles of the amplitude $\omega_{1}(t)$ and the frequency $\Delta \omega_{1}(t)$ of an HS pulse $g_{1}(t)$ for $\mu=5$. (b) Fourier transformation $G_{1}(\omega)$ of $g_{1}(t)$.

where the origin of time, namely, $t=0$, is taken at the middle of the HS pulse, $\omega_{\max }$ is the maximum amplitude in the HS pulse, and $\mu$ and $\beta$ are real constants. In practice, the HS pulse is implemented by generating a radio-frequency (rf) signal with amplitude

$$
\omega_{1}(t)=\omega_{\max } \operatorname{sech}(\beta t)
$$

and frequency

$$
\Delta \omega(t)=\mu \beta \tanh (\beta t)
$$

Since tanh ranges from -1 to 1 , the frequency is swept over $2 \mu \beta$ by the HS pulse. Alternatively, frequency sweep can be implemented through modulation of the phase $\phi$ of the rf signal according to

$$
\phi(t)=\int d t \Delta \omega(t)=-\mu \ln (\operatorname{sech}(\beta t))
$$

Derivation of the above equations involving the hyperbolic functions is described in the supplementary information.

We consider an HS pulse with an excitation bandwidth set to $1 / n$ of the probe bandwidth $\Delta \Omega$, namely, to $\Delta \Omega / n$. The profile of the efficiency of pulse excitation as a function of the frequency is characterized by a function $G_{1}(\omega)=\mathrm{F}\left[g_{1}(t)\right]$, where $\mathrm{F}$ denotes Fourier transformation. As an example, Fig. 1(a) and (b) show $g_{1}(t)$ and $G_{1}(\omega)$ for $\mu=5$.

Now, we apply comb modulation by multiplying $g_{1}(t)$ with a comb function

$$
\delta_{T}(t)=\sum_{k=-\infty}^{\infty} \delta(t-k T)
$$




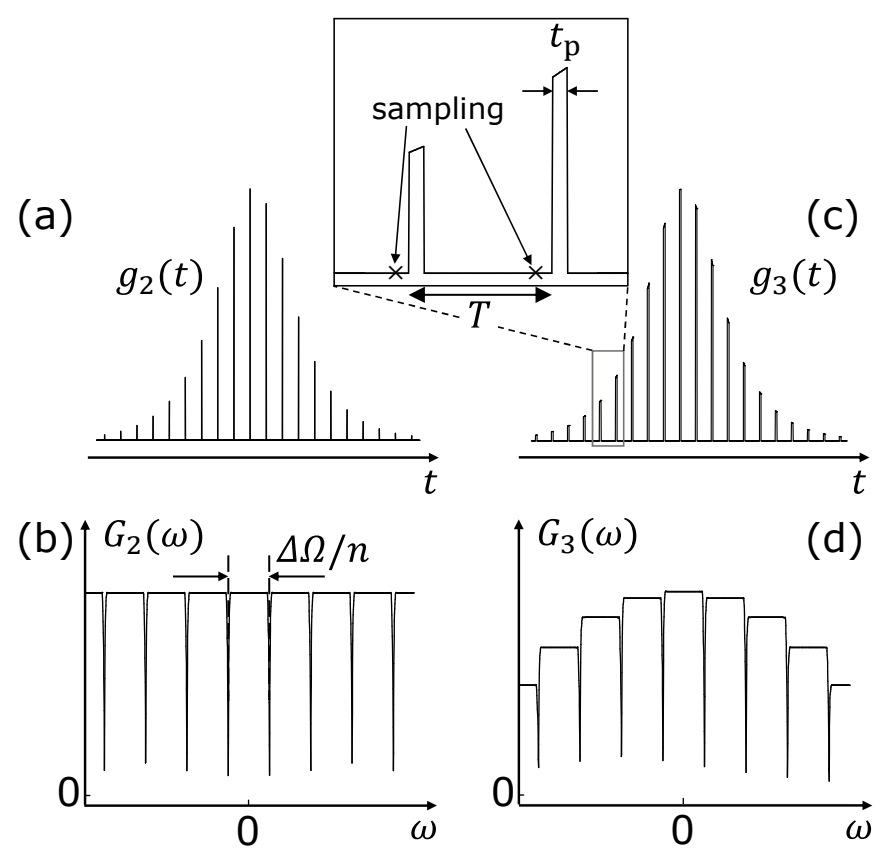

Figure 2: Profiles of (a) $g_{2}(t)$, (b) $G_{2}(\omega)$, (c) $g_{3}(t)$, and (d) $G_{3}(\omega)$.

The inset describes timings of data sampling.

obtaining

$$
g_{2}(t)=\delta_{T}(t) g_{1}(t)
$$

The time dependence of $g_{2}(t)$ is depicted in Fig. 2(a). The excitation profile $G_{2}(\omega)$ is represented by Fourier transforming $g_{2}(t)$ as

$$
\begin{aligned}
G_{2}(\omega) & =\mathrm{F}\left[g_{2}(t)\right]=\mathrm{F}\left[\delta_{T}(t) g_{1}(t)\right] \\
& =\mathrm{F}\left[\delta_{T}(t)\right] * \mathrm{~F}\left[g_{1}(t)\right]=\delta_{2 \pi / T}(\omega) * G_{1}(\omega),
\end{aligned}
$$

where the asterisk denotes convolution. Note that Fourier transformation of a comb function $\delta_{T}(t)$ in the time domain is also another comb function $\delta_{2 \pi / T}(\omega)$ in the frequency domain, and that of the HS function $g_{1}(t)$ shows a band-selective function $G_{1}(\omega)$. Since the Fourier transformation of a multiplication of a pair of functions is a convolution of the individually Fourier-transformed functions, we can see that the effect of comb modulation is to periodically replicate the original excitation profile in the frequency domain. If we choose the period $T$ of comb modulation in the time domain such that $2 \pi / T=\Delta \Omega / n$, the resultant excitation profile in the frequency domain covers a wide range, as shown in Fig. 2(b). 
In practice, the width of the modulation cannot be made infinitesimally short. To take account of the effect of the finite width $t_{\mathrm{p}}$ of modulation, we introduce a rectangular function $\sqcap\left(t / t_{\mathrm{p}}\right)$ and write a realistic modulation function as $\sqcap\left(t / t_{p}\right) * \delta_{T}(t)$. The excitation pulse $g_{3}(t)$ (Fig. 2(c)) and the profile $G_{3}(\omega)=\mathrm{F}\left[g_{3}(t)\right]$ (Fig. 2(d)) in the frequency domain are given by

$$
\begin{gathered}
g_{3}(t)=\left[\sqcap\left(t / t_{p}\right) * \delta_{T}(t)\right] \cdot g_{1}(t), \\
G_{3}(\omega)=\left[\operatorname{sinc}\left(\omega t_{p} / 2\right) \cdot \delta_{2 \pi / T}(\omega)\right] * G_{1}(\omega),
\end{gathered}
$$

respectively. As we can see in Fig. 2(d), the effect of the finite width is to scale the excitation profile according to the sinc function. The full width at half height of $\operatorname{sinc}(x)$ is ca. $2 \pi \cdot 0.6 \sim 3.8$. When we choose $t_{\mathrm{p}}$ such that the profile of $G_{3}(\omega)$ covers the probe bandwidth $\Delta \Omega, t_{\mathrm{p}}$ may be set to fulfill

$$
t_{\mathrm{p}} \leq \frac{8 \pi \cdot 0.3}{\Delta \Omega}
$$

\subsection{Coarse and fine surveys}

Let us suppose that the rapid-scan NQR signal is acquired in such a way that a single data point is sampled during each gap in the comb-modulated HS pulse, as described in the inset of Fig. 2(c). Then, the sampling interval is determined to be the period $T$ of comb modulation. According to the sampling theorem, the bandwidth of the acquired data is given by the inverse of sampling interval, namely, $2 \pi / T$ in units of angular frequency, which is now equal to $2 \pi / T=\Delta \Omega / n$. Thus, the window through which we observe the response from the spin system is narrower than the excitation bandwidth $\Delta \Omega$ by the factor $n$. Then, there can be such signal components that settle inside the excitation bandwidth but outside the detection bandwidth. Importantly, it does not mean we do not observe anything. The effect, well known as aliasing, is to bring such signals into view, with their Fourier component folded back and forth until they settle within the detection bandwidth. In many applications of magnetic resonance, signal aliasing is unwelcome and avoided because of the ambiguity of the resonance frequency. Nevertheless, in the coarse survey of RASGADO NQR, the very existence, or the absence, of the resonance within the current excitation band can be important information, and be exploited in 
the search of unknown, sparse resonances, where a wide range of frequencies needs to be covered.

Then, what is required toward unique determination of the resonance frequency is to perform another measurement in a different run with a shorter sampling interval. Upon detection of the resonance, we are now well informed about at which moment during the application of the gapped rapid scan the transverse magnetization becomes the maximum. Thus, we propose to run the identical sequence in the first part of the sequence until the moment when the rapid scan gave the maximum signal amplitude. What we need to do then is simply to quit the comb-modulated HS pulse on its way, and immediately start acquiring the free induction decay (FID). Now that the transmitter has been switched off, there is no restriction on the data-sampling rate $t_{\mathrm{dw}}$, which is set short enough to make the acquisition bandwidth $2 \pi / t_{\mathrm{dw}}$ cover the excitation bandwidth $\Delta \Omega$. In this way, we can observe the signal without aliasing and determine the NQR frequency in the fine survey mode.

\subsection{Sensitivity and Experimental Time}

The magnitude of the transverse magnetization excited by the rapid-scan scheme is comparable to that created by a $\pi / 2$ pulse, so that the difference in the amplitudes of the NQR signal obtained with a single shot of both sequences is not significant. Then, in the case of the known resonance frequency, the sensitivity attainable within a given experimental time is determined by how frequent can the sequences be implemented and the signal be accumulated. According to Gupta et al., the comparison of the expected signal-to-noise ratio $(S / N)_{\mathrm{p}}$ in the pulse method with that $(S / N)_{\mathrm{rs}}$ in the rapid-scan method is given by[3]

$$
\frac{(S / N)_{\mathrm{p}}}{(S / N)_{\mathrm{rs}}}=\left[\frac{T_{\mathrm{rs}}\left(1-e^{-T_{\mathrm{p}} / T_{1}}\right)\left(1+e^{-T_{\mathrm{rs}} / T_{1}}\right)}{T_{\mathrm{p}}\left(1+e^{-T_{\mathrm{p}} / T_{1}}\right)\left(1-e^{-T_{\mathrm{rs}} / T_{1}}\right)}\right]^{\frac{1}{2}},
$$

where $T_{\mathrm{p}}$ and $T_{\mathrm{rs}}$ are the repetition-time intervals of the sequences in the pulse and the rapid-scan methods, respectively, and $T_{1}$ is the spin-lattice relaxation time. When $T_{1}$ is considerably longer than both the dephasing time $T_{2}^{*}$ and the time consumed by rapid scan, the attainable sensitivities are nearly identical[3]. 
Conversely, when the resonance frequency is unknown and has to be sought over a wide spectral range, blank applications of excitation with no response from the spin system cannot be avoided until the excitation band happens to include the resonance frequency. Now, it is necessary to take account of the cost of such blank shots in the evaluation of the expected sensitivity attainable within a given experimental time. To appreciate the advantage of the RASGADO NQR approach in this respect, let us consider the time $T_{\text {coarse }}$ consumed by the coarse survey

$$
T_{\text {coarse }}=\left(T_{\mathrm{s}}+T_{\mathrm{rs}}\right) \cdot M \cdot N
$$

and that $T_{\text {fine }}$ of the fine survey

$$
T_{\text {fine }}=\left(T_{\mathrm{s}}^{\prime}+T_{\mathrm{a}}+T_{\mathrm{rs}}\right) \cdot N \text {. }
$$

Here, $T_{\mathrm{s}}$ is the width of the HS pulse, $N$ is the number of signal acquisitions, and $M$ is the number of spectral regions we perform survey over. A different symbol $T_{\mathrm{s}}^{\prime}\left(<T_{\mathrm{s}}\right)$ is given for the excitation width in the fine survey, as the HS excitation is aborted during its application, and the additional $T_{\mathrm{a}}$ represents the time required for acquiring the FID. Here, we assume that the signal is found in one of $M$ spectral regions. The overall time required in RASGADO NQR is straightforwardly obtained as

$$
\begin{aligned}
T_{\mathrm{RASGADO}} & =T_{\text {coarse }}+T_{\text {fine }} \\
& =\left[\left(T_{\mathrm{s}}+T_{\mathrm{rs}}\right) M+T_{\mathrm{s}}^{\prime}+T_{\mathrm{a}}+T_{\mathrm{rs}}\right] \cdot N .
\end{aligned}
$$

Since the repetition delay $T_{\mathrm{rs}}$ needs to be a few times the spin-lattice relaxation time $T_{1}$, which is often much longer than the other time parameters,

$$
T_{\mathrm{RASGADO}} \sim T_{\mathrm{rs}}(M+1) N
$$

In the traditional pulse method with frequency-stepping, the experimental time for surveying the range of $\Delta \Omega$ is

$$
\begin{aligned}
T_{\text {pulse }} & =\left(T_{\mathrm{a}}+T_{\mathrm{p}}\right) \cdot M \cdot N \cdot \frac{\Delta \Omega}{\Delta \omega_{\mathrm{ex}}} \\
& \sim T_{\mathrm{p}} \cdot M \cdot N \cdot \frac{\Delta \Omega}{\Delta \omega_{\mathrm{ex}}}
\end{aligned}
$$


where $\Delta \omega_{\text {ex }}$ is the effective excitation range of the pulse. To survey the range of $\Delta \Omega$ by the traditional method, one needs to repeat the experiment $\Delta \Omega / \Delta \omega_{\text {ex }}$ times. Now, the ratio of the required experimental times required to collect the data with $N$ signal accumulations for the two methods is

$$
\frac{T_{\mathrm{RASGADO}}}{T_{\mathrm{pulse}}} \approx \frac{T_{\mathrm{rs}}(M+1) N}{T_{\mathrm{p}} \cdot M \cdot N \cdot \frac{\Delta \Omega}{\Delta \omega_{\mathrm{ex}}}} \approx \frac{\Delta \omega_{\mathrm{ex}}}{\Delta \Omega}
$$

Since the excitation bandwidth $\Delta \omega_{\text {ex }}$ is in general much narrower than the circuit bandwidth $\Delta \Omega$, the RASGADO scheme consumes much shorter experimental time for a given accumulation number. In other words, within a given experimental time, the meaningful signal can be accumulated over much larger number of times, and the sensitivity can be significantly higher for the proposed scheme.

It is worth pointing out that the ISS scheme also offers a very efficient way in the search for NQR signals[2]. This is indeed true when the circuit bandwidth can be made much larger than the typically attainable values by developing special hardware[1].

\section{Spin dynamics under comb-modulated HS pulse}

\subsection{HS pulses without gaps}

To evaluate the dynamics of the nulcear magnetization during application of the combmodulated HS pulse, we carried out simulations by numerically solving the Bloch equation using the 4th order Runge-Kutta method. Figure 3 shows simulated trajectories of magnetization $\mathbf{M}$, initially in thermal equilibrium $\left(0,0, M_{0}\right)$, and under application of HS pulses with various frequency-sweep rates. The results depicted here are well known. That is, for sufficiently slow passage such that the adiabatic condition[9],

$$
\left|\frac{d \Delta \omega}{d t}\right| \ll \frac{\gamma H_{\mathrm{e}}^{2}}{\sin \theta}
$$

where $\gamma$ is the gyromagnetic ratio, $H_{\mathrm{e}}$ is amplitude of the effective field and $\theta$ is the angle of the effective field with respect to the $z$ axis, is satisfied, the magnetization follows the effective field (Fig. 3(a)), which, in the present case, is initially far-off resonance and thereby nearly parallel to the $z$ axis, then gradually tilts towards the $x y$ plane as it 
approaches on-resonance, and after passing through the resonance, goes far-off resonance to the opposite direction anti-parallel to the $z$ axis.
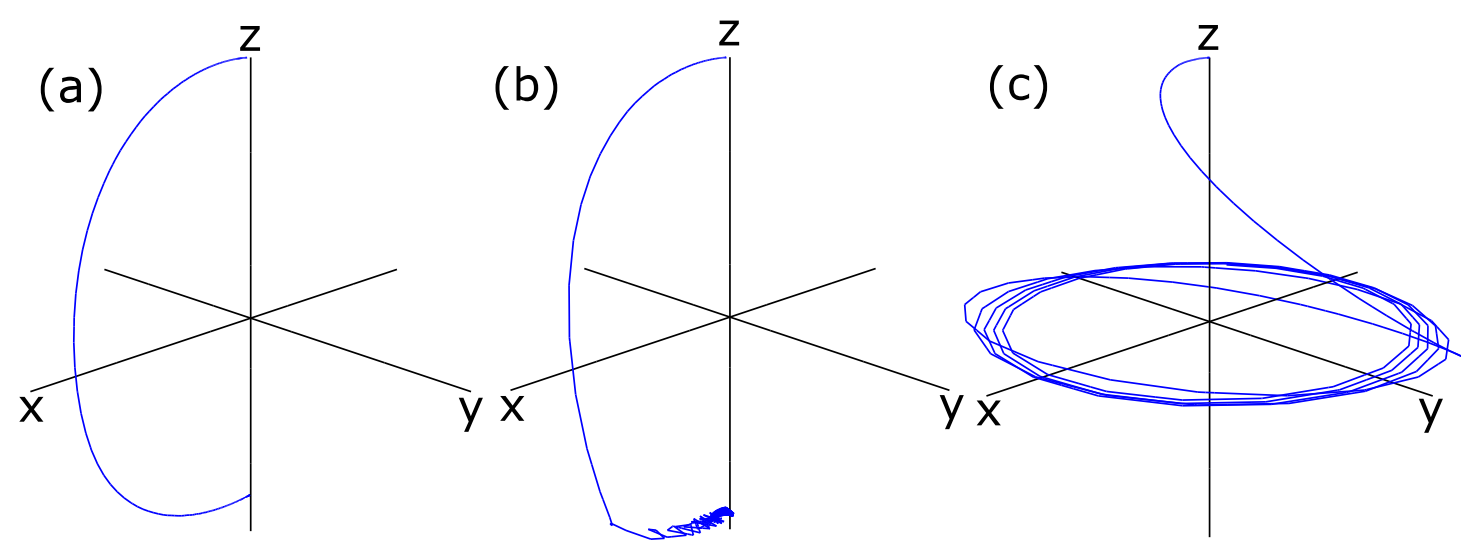

Figure 3: Simulated trajectories of the magnetization $\mathbf{M}$ during the HS pulse with $\omega_{\max }=2 \pi \cdot 6 \mathrm{kHz}$ and $\mu \beta=2 \pi \cdot 25 \mathrm{kHz}$. The pulse duration was (a) $10 \mathrm{~ms}$, (b) $8 \mathrm{~ms}$, and (c) $5 \mathrm{~ms}$. $\mathbf{M}$ was initially set to $\left(0,0, M_{0}\right)$, and allowed to evolve according to the Bloch equation.

As increasing the rate of frequency sweep, or as decreasing the amplitude of the applied rf pulse, the adiabatic condition begins to become strict in particular when the effective field is passing through the region close to the resonance. In Fig. 3(b), the magnetization barely follows the trajectory of the effective field at the end of the sweep. As the sweep rate is further increased, the magnetization will no longer be able to follow the effective field (Fig. 3(c)), and the projection of the three-dimensional trajectory of the magnetization onto the $x y$ plane provides a rapid-scan signal.

The rapid-scan signal reflects the linear response of the spin system to the excitation, which, in the present case, is the HS pulse. Therefore, it carries the information required to reproduce the response of the spin system to any excitation. In particular, the impulse response or its Fourier-transformed counterpart is of interest, because the former is a similar figure to the FID following an intense pulse excitation, and hence the latter gives the NMR/NQR spectrum in the conventional sense.

In some cases, it is possible to simultaneously acquire nuclear induction signals while the rapid-scan excitation is applied. Indeed, the pioneering work on rapid-scan NMR 
demonstrated by Gupta et al.[3] used a continuous-wave NMR spectrometer to perform rapid magnetic-field sweep with a frequency-fixed rf irradiation, so that the excitation signal leaking into the receiving circuit can be compensated straightforwardly. In applications of continuous-wave rapid scan to MRI reported by Idiyatullin et al.[10], frequency sweep was adopted in a fixed magnetic field, whose option is natural and straightforward for modern NMR systems equipped with a superconducting magnet. Since the characteristics of the electrical circuit now depends on the frequency, the rapid-scan MRI signal is accompanied with the frequency-dependent leakage of the excitation signal. When the amplitude of the former is sufficiently large, the latter could be eliminated by post data processing. However, when one deals with such small NMR signals that one needs to increase the gain of the receiver to such an extent that the leakage saturates the receiver, one can no longer resort to simultaneous excitation and acquisition and needs to adopt the time-sharing scheme by making periodic gaps in the excitation pulse to acquire the signal.

According to Idiyatullin et al.[6], who reported in-depth SWIFT studies on the effect of the gap inserted into rapid-scan HS pulses as well as the effect of digitization of shaped pulses, the gaps made in the HS pulse do not affect the bandwidth of excitation, but decreases the flip angle, and the scaling factor is proportional to the duty ratio of the gapped excitation pulse. It follows that, in order to reproduce the same spin dynamics by the gapped pulse as that by the continuous gapless pulse, one needs to increase the amplitude of the excitation pulse.

In practice, the width of the gap for data sampling cannot be made arbitrarily short, because one need to wait after the individual sub-pulses for at least the dead time, i.e., the time required for the pulse to decay below the noise floor of the receiver before the signal can become acquired. In the following, we examine the gapped rapid-scan NMR signals for various excitation and detection bandwidths.

\section{2 comb-modulated HS pulses}

Figure 4 shows a trajectory of the magnetization under application of a gapped HS pulse with comb modulation, to be used in the RASGADO scheme. Here, the modulation 


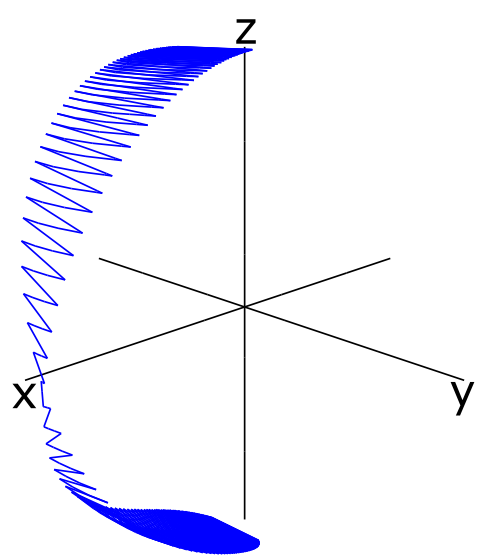

Figure 4: A simulated trajectory of the magnetization, initially $\left(0,0, M_{0}\right)$, and under application of a comb-modulated HS pulse with $\omega_{\max }=2 \pi \cdot 60 \mathrm{kHz}, \mu \beta=2 \pi \cdot 25 \mathrm{kHz}$, finite pulse width of $t_{\mathrm{p}}=2 \mu \mathrm{s}$, and period of $20 \mu \mathrm{s}$. The duration of the pulse was 10 ms.

period $T$ and the width $t_{\mathrm{p}}$ of the subpulses were set to $20 \mu \mathrm{s}$ and $2 \mu \mathrm{s}$, respectively, so that the duty ratio was $2 / 20=0.1$. The HS pulse with a bandwidth of $2 \pi \cdot 50 \mathrm{kHz}$ and duration of $10 \mathrm{~ms}$ was applied. The intensity $\omega_{\max }$ of the excitation pulse was set to $2 \pi \cdot 60 \mathrm{kHz}$, which is 10 times (equal to the inverse of the duty ratio of 0.1 ) that in the case of Fig. 3, in which the response of the spin system to the gapless excitation was simulated. As demonstrated, the overall profiles of the trajectories for the gapped and gapless excitations were similar to each other. The zigzag profile seen in the case of the gapped case is a consequence of nutation about the effective field for $2 \mu$ s and off-resonance precession for $18 \mu \mathrm{s}$, repeated one after another.

Figure 5 shows trajectories when the centers of frequency sweep are different. Fig. 5 (a) shows that the trajectory (blue line) and the sampling points (red dots) of the magnetization during a comb-modulated HS pulse whose duty ratio and sweep time were the same as those of Fig. 4 , while $\omega_{\max }$ was set to $2 \pi \cdot 15 \mathrm{kHz}$. Now, the adiabatic condition is violated, and the trajectory of the magnetization carries the linear response of the spin system. Fig. 5 (b) shows the trajectories when the offset of the center frequency of the sweep from the resonant frequency was $2 \pi \cdot 50 \mathrm{kHz}$, while the other conditions were unchanged from those used to obtain the data shown in Fig. 5(a). We see that the 

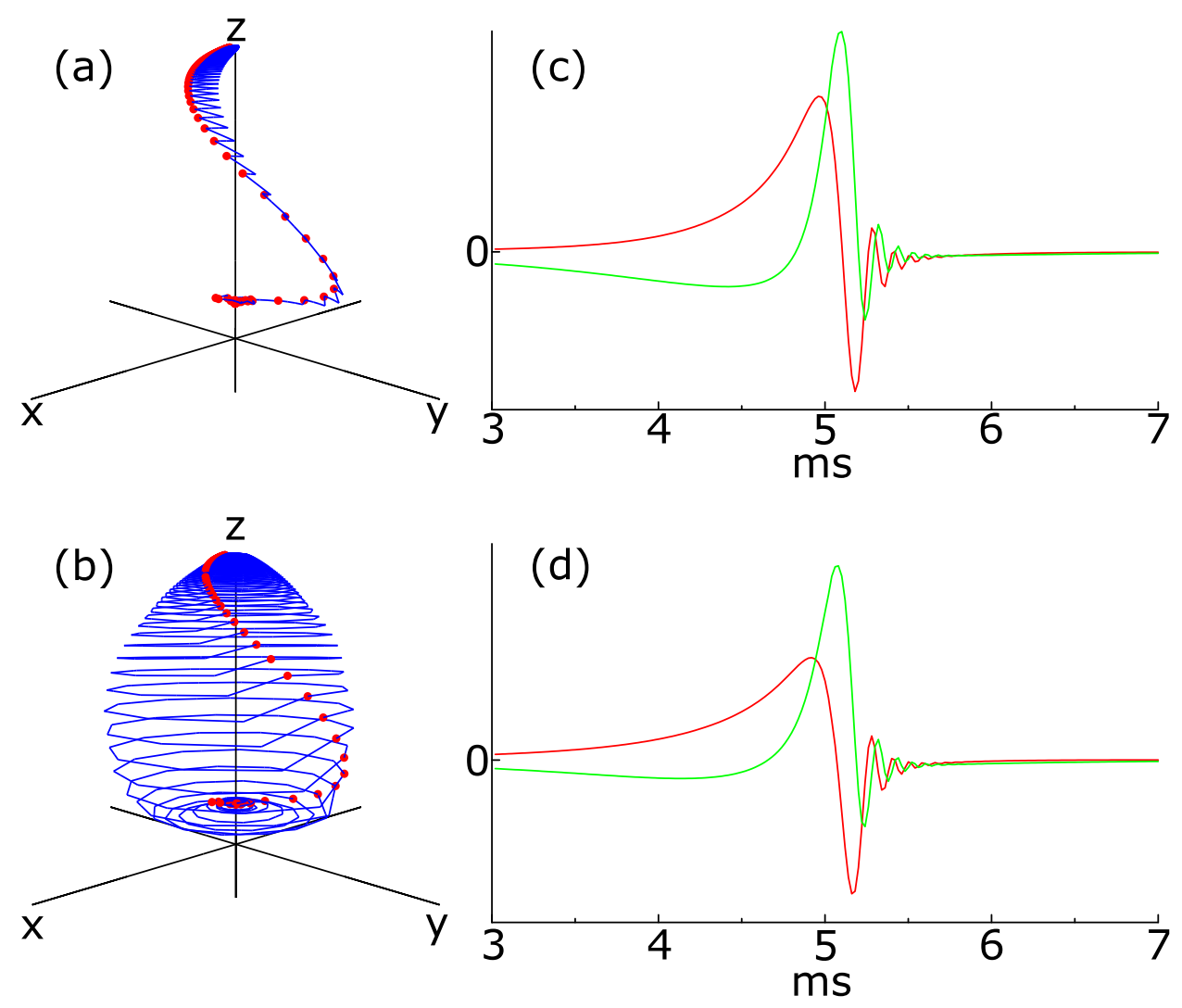

Figure 5: (a)(b) Simulated trajectories of the magnetization (blue lines) and the sampling points (red dots) under application of a comb-modulated HS pulse with $\omega_{\max }=2 \pi \cdot 15 \mathrm{kHz}, \mu \beta=2 \pi \cdot 25$ $\mathrm{kHz}$. The center frequency was set on-resonance in (a), whereas the frequency offset was $2 \pi \cdot 50 \mathrm{kHz}$ in (b), and the sampling interval $T$ was set equal to the gap interval $t_{\mathrm{dw}}=20 \mu \mathrm{s}$. (c)(d) Corresponding in-phase (red) and quadrature (green) components of the expected NQR signals obtained from the projection of the magnetization vector onto the $x y$ plane. 
magnetization undergoes a full revolution during the gap of the comb-modulated excitation. Even though the simulated trajectories were such different, the apparent signals that would be obtained with the sampling-time interval $T$ became quite similar, as indicated by the red dots in Fig. 5(b). Fig. 5(c) and (d) show the in-phase and quadrature components of the rapid scan signal obtained from the projection of the magnetization vector onto the $x y$ plane.

\section{Experimental}

In this work, we aimed at demonstrating RASGADO NQR of ${ }^{35} \mathrm{Cl}$ in potassium chlorate $\left(\mathrm{KClO}_{3}\right)$, and of ${ }^{63} \mathrm{Cu}$ and ${ }^{65} \mathrm{Cu}$ in copper oxide $\left(\mathrm{Cu}_{2} \mathrm{O}\right)$, whose resonant frequencies are known to be $2 \pi \cdot 28.953 \mathrm{MHz}[11], 2 \pi \cdot 26.704 \mathrm{MHz}[12]$ and $2 \pi \cdot 24.714 \mathrm{MHz}[12]$, respectively. The spin quantum number is $3 / 2$ for all of these isotopes, so that they resonate at a single frequency.

We built an NQR probe by winding a coil with a diameter of $12 \mathrm{~mm}$, length of 12 $\mathrm{mm}$, and number of turns of 5.5. A tank circuit was assembled with a pair of trimmer capacitors, so that the circuit could be impedance-matched at frequencies ranging from $2 \pi \cdot 23.08$ to $2 \pi \cdot 32.37 \mathrm{MHz}$. The bandwidth of the resonant circuit $\Delta \Omega$ was ca. $2 \pi \cdot 500$ $\mathrm{kHz}$ at $2 \pi \cdot 28.953 \mathrm{MHz}$ and ca. $2 \pi \cdot 400 \mathrm{kHz}$ at frequencies from $2 \pi \cdot 24.7 \mathrm{MHz}$ to $2 \pi \cdot 26.7$ MHz. An Opencore NMR spectrometer[13] was used for pulse transmission and signal reception.

In the coarse survey, the spin system was excited by an HS pulse with comb modulation with a period $T$ of $20 \mu \mathrm{s}$, pulse length $t_{\mathrm{p}}$ of $2 \mu \mathrm{s}$ and gap of $18 \mu \mathrm{s}$. The range of frequency sweep in the original HS pulse, before application of comb modulation, was set to $2 \pi \cdot 50$ $\mathrm{kHz}$. The number of sampling points was 500. In the fine survey, the sampling interval and sampling points were set to $1 \mu$ s and 2048, respectively. The repetition time interval $T_{\mathrm{rs}}$ was set to $1 \mathrm{~s}$. All NQR measurement were carried out at $77 \mathrm{~K}$ by immersing the sample together with the probe in liquid nitrogen. 


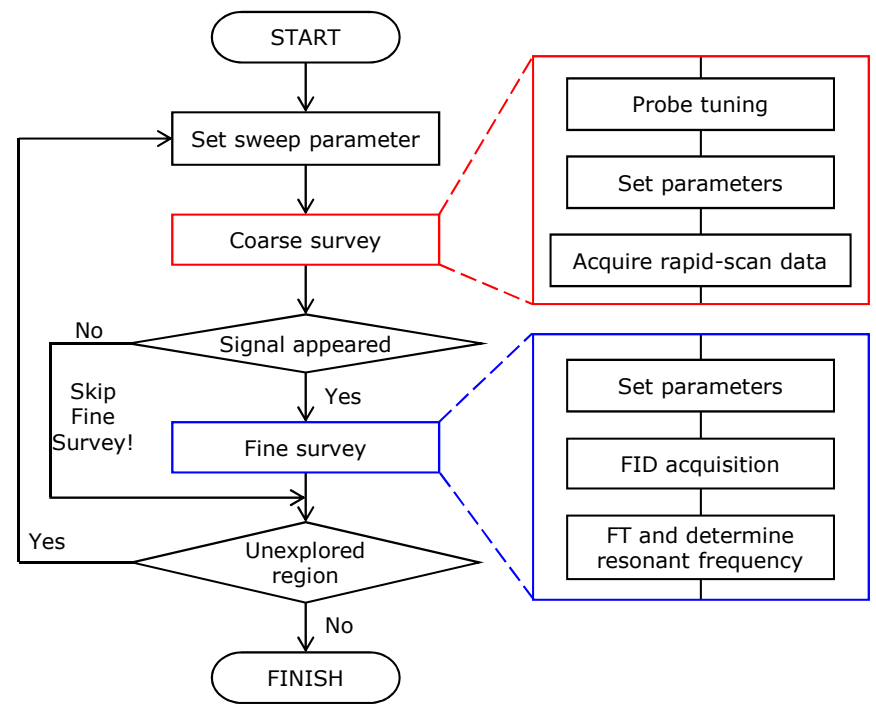

Figure 6: A flowchart of RASGADO NQR.

\subsection{Protocol of RASGADO NQR}

Figure 6 shows a flowchart of implementing RASGADO NQR. Let us divide the full spectral range over which the resonance is sought into segments with such a width that fits in the bandwidth $\Delta \Omega$ of the tank circuit of the NQR probe. The coarse surveys are performed to the individual spectral regions separately in the following way. The first step of each coarse survey is to adjust the trimmer capacitors of the probe circuit to attain impedance matching at the center frequency of the current spectral region. Then, the comb-modulated HS pulse, such that its excitation profile well covers the probe bandwidth as discussed in Section 1, is applied. The gaps that arise during application of the comb-modulated HS pulse serve for the windows of data sampling. In the case that the NQR frequency is within the excitation bandwidth, the transverse magnetization ought to develop, so that the acquired data would show a signal with a figure similar to that would be expected in the conventional rapid-scan experiments. If this is the case, we inspect the data obtained in the coarse survey, extracting the time at which the rapidscan signal exhibited the largest amplitude, and then proceeding to the fine survey mode. Otherwise, we skip the fine survey, and perform the coarse survey in the next spectral region of interest.

The excitation applied in the beginning of the fine survey mode is exactly identical to that of the coarse survey. That is, we start with applying the comb-modulated HS pulse, 
with no change in the excitation parameters. Then, we abruptly pause and abort the implementation of the comb-modulated HS pulse; now that we have just switched from the coarse survey mode, we ought to be already equipped with the knowledge of the moment when the transverse magnetization becomes the maximum. As soon as the excitation has been aborted, we begin acquisition of the induction signal caused by free precession of the transverse magnetization. Now the transmitter is switched off throughout the acquisition time, we sample the data at a sufficiently fast rate, making sure that the detection bandwidth in the fine survey mode well covers the probe bandwidth. The NQR spectrum is obtained by Fourier transforming the acquired FID, and the NQR frequency is finally determined from the position of the resonance line appearing in the spectrum.

\section{Results and discussion}

Figure 7 (a) and (b) show rapid scan signals obtained with the coarse survey where the offsets of frequency sweep center from the resonant frequency were 0 and $2 \pi \cdot 50 \mathrm{kHz}$. The apparent behavior of the signal in Fig. 7 (b) was almost the same as that in Fig. 7(a), because of the signal aliasing.

Next, the same sequence was implemented and aborted when the rapid scan signal was the largest, then FID acquisition was started immediately with a sampling interval $t_{\mathrm{dw}}$ of $1 \mu \mathrm{s}$, which was sufficiently short. As shown in Fig. 7 (c) and (d), we obtained the FID signals that reflect the accurate information of the resonant frequency.

Fig. 7 (e) and (f) are the NQR spectra obtained from deconvolution of the rapid-scan data shown in Fig. 7 (a) and (b), according to the procedure described by Gupta[3], and from Fourier transformation of the FID signals shown in Fig. 7 (c) and (d). For zero frequency offset, the NQR lines appeared at same position, while for the frequency offset of $2 \pi \cdot 50 \mathrm{kHz}$, the NQR peak obtained from rapid scan did not reflect the correct resonant frequency due to signal aliasing. Conversely, the NQR spectrum obtained from the FID acquired in the fine survey mode allowed to correct determination of the resonant frequencies for both experiments. The rapid-scan signals and the FID signals in the fine surveys obtained for various frequency offsets are summarized in the supplementary information. Figure 8 shows spectra obtained by the fine survey when the frequency 

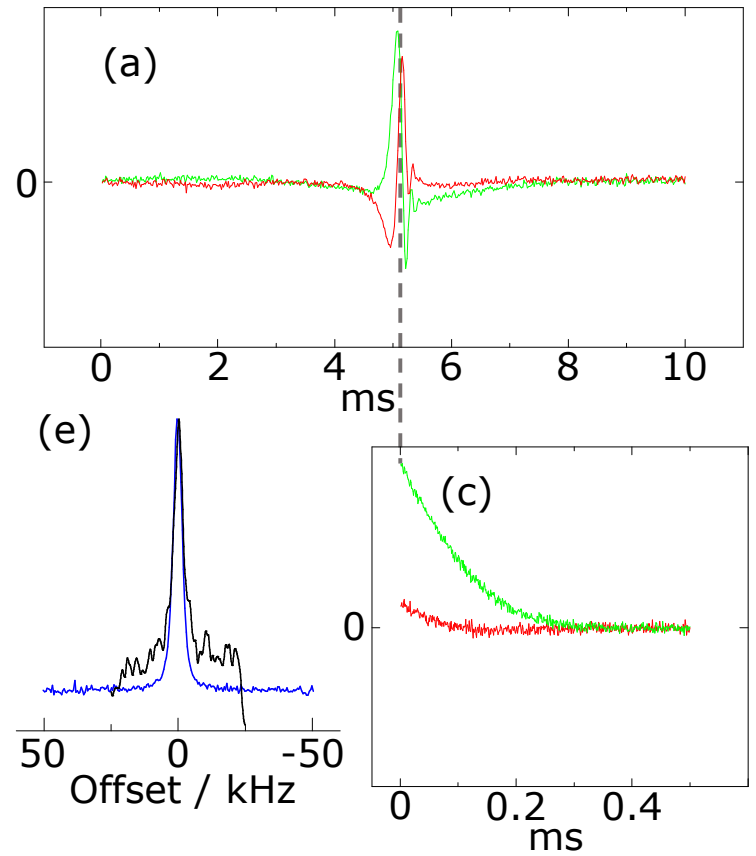
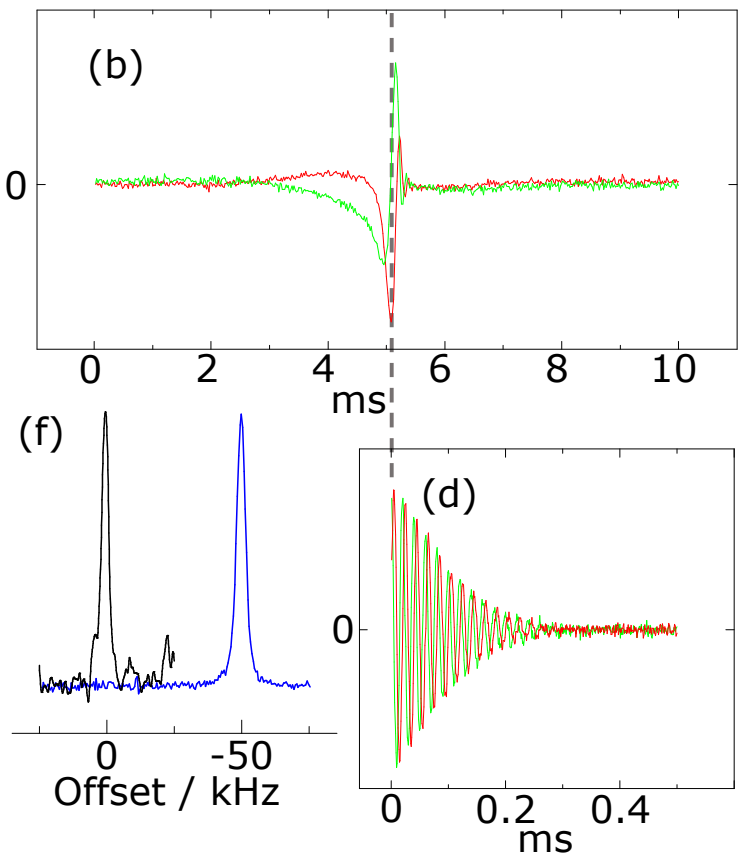

Figure 7: Experimental rapid-scan ${ }^{35} \mathrm{Cl} \mathrm{NQR}$ signals of $\mathrm{KClO}_{3}$ with frequency offset of (a) $0 \mathrm{kHz}$ and (b) $2 \pi \cdot 50 \mathrm{kHz}$ obtained in the coarse survey mode by acquiring the signal during the gaps of the comb-modulated HS excitation. Red and green lines describe in-phase and quadrature components, respectively. (c)(d) Corresponding FID signals obtained in the fine survey mode by aborting the rapid scan at $t=5.1 \mathrm{~ms}$ (indicated by vertical broken lines) and then immediately starting acquisition with a dwell time of 1 $\mu$ s. (e)(f) NQR spectra obtained from deconvolution of the rapidscan data (a)(b) (black lines) and from Fourier transformation of the fine survey data (c)(d) (blue lines). 


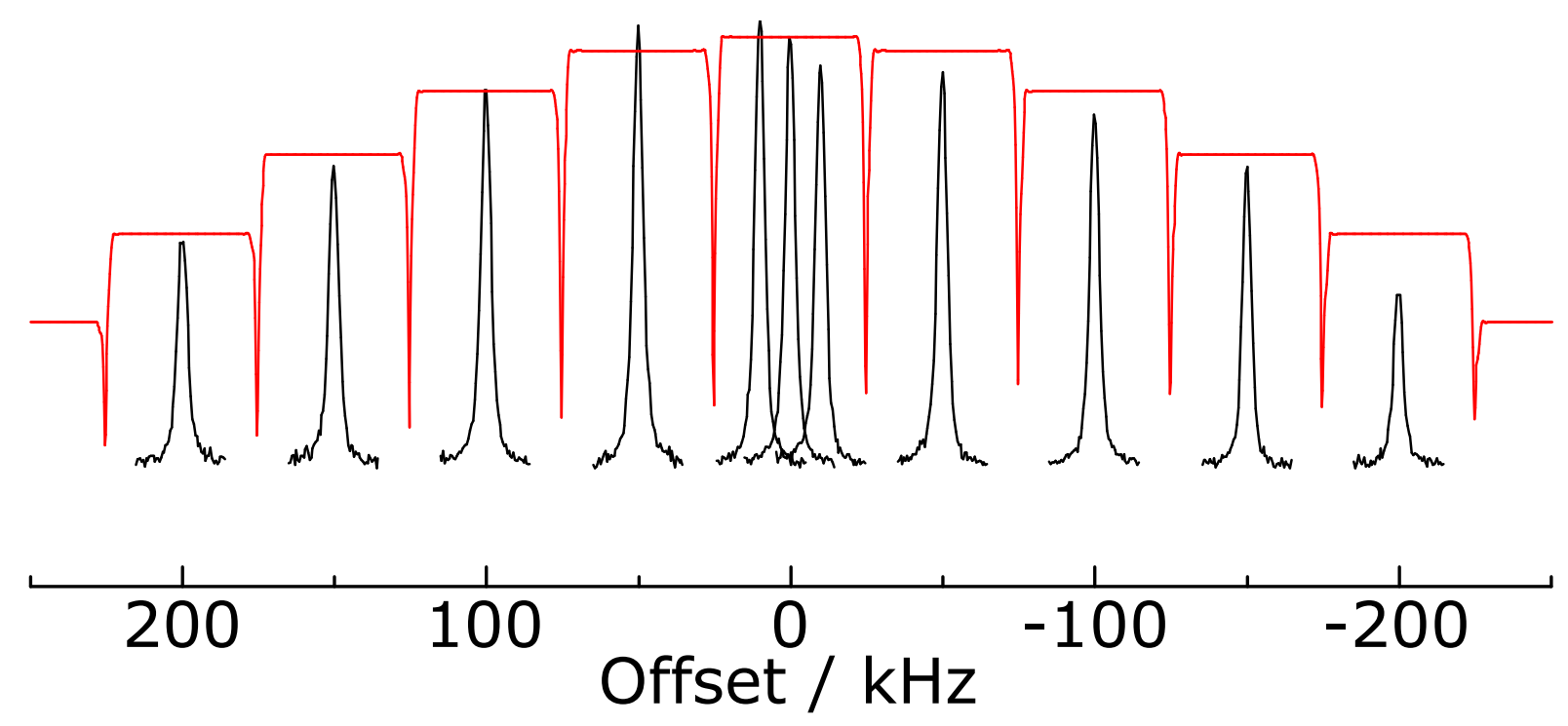

Figure 8: ${ }^{35} \mathrm{Cl} \mathrm{NQR}$ spectra of $\mathrm{KClO}_{3}$ obtained by the fine survey with frequency offsets of $0, \pm 2 \pi \cdot 10, \pm 2 \pi \cdot 50, \pm 2 \pi \cdot 100, \pm 2 \pi \cdot 150$, and $\pm 2 \pi \cdot 200 \mathrm{kHz}$.

offsets were $0, \pm 2 \pi \cdot 10, \pm 2 \pi \cdot 50, \pm 2 \pi \cdot 100, \pm 2 \pi \cdot 150$ and $\pm 2 \pi \cdot 200 \mathrm{kHz}$ from the resonance frequency. The signal intensity decreased as increasing the offset, showing the profile that corresponds to the sinc function, as described in Fig. 2(d).

In ${ }^{63} \mathrm{Cu}$ and ${ }^{65} \mathrm{Cu}$ NQR measurements of $\mathrm{Cu}_{2} \mathrm{O}$, we explored the signals over frequencies ranging from $2 \pi \cdot 24.0$ to $2 \pi \cdot 27.2 \mathrm{MHz}$. As the probe bandwidth was $2 \pi \cdot 400 \mathrm{kHz}, 8$ separate coarse surveys were performed by setting the center frequencies of the HS pulse to $2 \pi \cdot(24.2+0.4 m) \mathrm{MHz}(m=0,1, \ldots, 7)$, and adjusting probe tuning. The result was that the signals were detected for the case of $m=1$ and 6 in the coarse survey. Accordingly, we carried out the fine surveys for these two spectral regions, skipping the others.

Figure 9 shows the NQR spectra obtained by the Fourier transformation of FID signals acquired in the fine survey. The resonant frequencies were $2 \pi \cdot 26.706 \mathrm{MHz}$ and $2 \pi \cdot 24.713$ $\mathrm{MHz}$ and the ratio of signal intensity was 2:1. From the fact that the natural abundance of ${ }^{63} \mathrm{Cu}$ and ${ }^{65} \mathrm{Cu}$ are $69.17 \%$ and $30.83 \%$, and quadrupolar moment of them are -22.0 $\mathrm{fm}^{2}$ and $-20.4 \mathrm{fm}^{2}[14]$, we assigned the former resonant frequency to ${ }^{63} \mathrm{Cu}$, and the latter to ${ }^{65} \mathrm{Cu}$.

When one tries to search the ${ }^{63} \mathrm{Cu}$ and ${ }^{65} \mathrm{Cu}$ NQR frequencies over the frequency range 


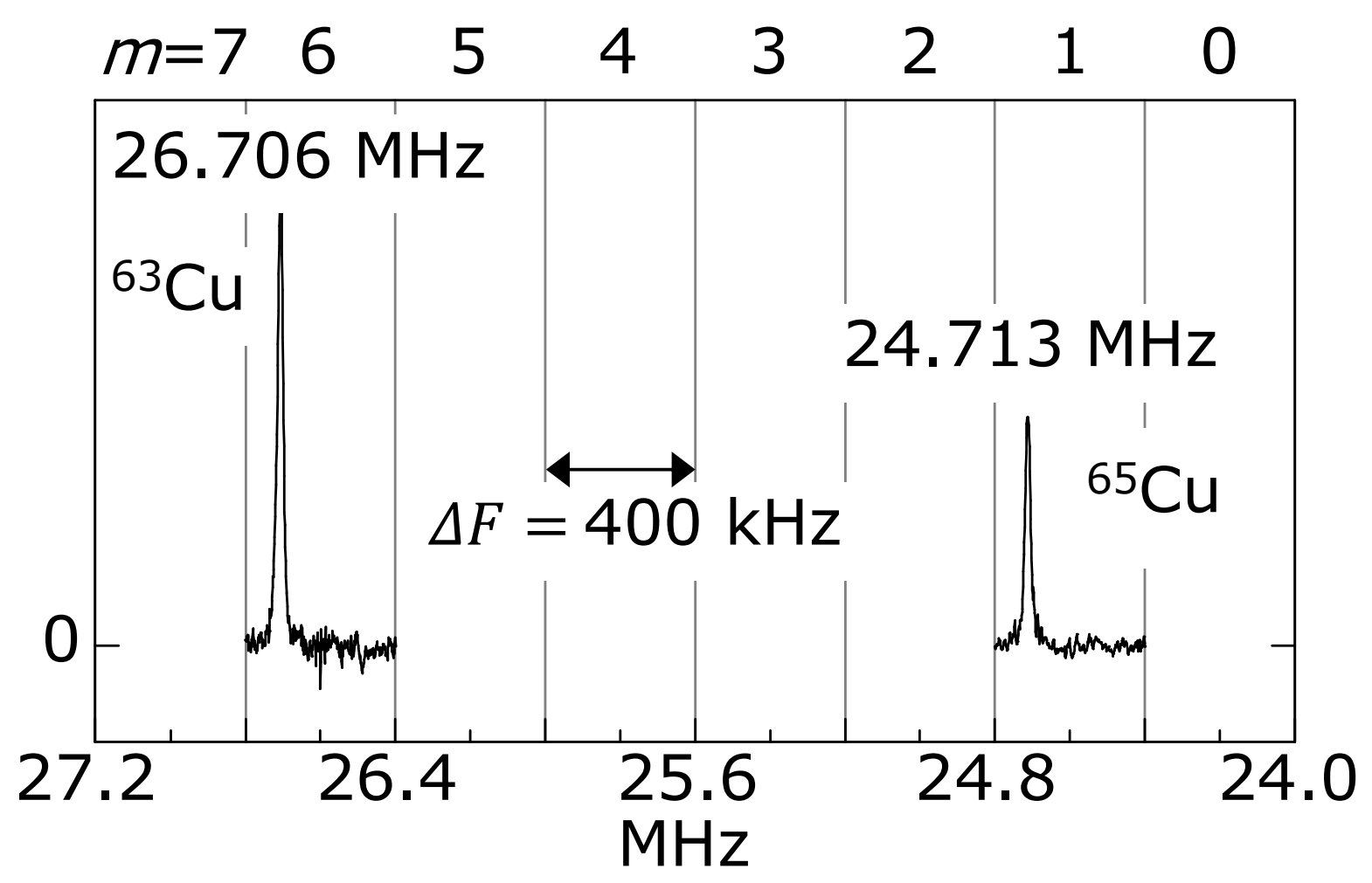

Figure 9: ${ }^{63} \mathrm{Cu}$ and ${ }^{65} \mathrm{Cu}$ NQR spectra in $\mathrm{Cu}_{2} \mathrm{O}$ obtained by the RASGADO method. 
of $2 \pi \cdot 2.8 \mathrm{MHz}$ by the conventional frequency-stepping method or ISS using the pulse with the same excitation bandwidth as that for the original (ungapped) HS pulse, namely, $2 \pi \cdot 50 \mathrm{kHz}$, one needs to perform 56 separate experiments with a frequency increment of $2 \pi \cdot 50 \mathrm{kHz}$. In the RASGADO scheme demonstrated in this work, the individual coarse survey covers the excitation band of as large as $2 \pi \cdot 400 \mathrm{kHz}$, thanks to the comb modulation. Thus, the number of the coarse survey was only 7 , out of which the two regions revealed the existence of the NQR signals. It follows that the fine surveys were performed only in these two regions, and as a consequence, 9 separate implementation (7 coarse, 2 fine) allowed determination of the ${ }^{63} \mathrm{Cu}$ and ${ }^{65} \mathrm{Cu}$ resonances.

\section{Conclusion}

The comb-modulated pulses offer wideband excitation and can be utilized in the search for NQR resonances. The periodic gaps in the comb-modulated pulse during which the transmitter is switched off serve for the data-sampling windows. Even though the acquisition bandwidth is limited by the inverse of the gap interval and is not sufficient to cover the entire excitation band, the signal, if present, would be aliased into view, and therefore one can at least tell the existence of the resonance within the extended excitation band of the comb-modulated pulses.

Upon finding such signs of the NQR signals, the identical comb-modulated HS pulse is partially applied until the moment when the transverse magnetization was found to become the maximum, and then, rf irradiation is aborted and acquisition of the FID signal is started with such a short sampling interval that covers the whole excitation range. Importantly, such experimental-mode switching is necessary only when the sign of the resonance is found during the coarse data sampling at the gaps of the comb excitation pulse. Since the resonance lines usually appear in a restricted region of the whole spectral range that is surveyed, the approach proposed here would be beneficial for acquiring NQR spectra in materials yet to be characterized. 


\section{Conflicts of interest}

There are no conflicts to declare.

\section{Acknowledgements}

This work was supported by JSPS Grant-in-Aid for Scientific Research (B) (Grant Number 18H01941), Innovation Areas "Mixed anion" (Grant Number 16H06440), and "Interface IONICS" (Grant Number JP19H05814).

\section{References}

[1] Hermann Scharfetter. An electronically tuned wideband probehead for NQR spectroscopy in the VHF range. Journal of Magnetic Resonance, 271:90-98, 2016.

[2] Hermann Scharfetter, Markus Bödenler, and Dominik Narnhofer. A cryostatic, fast scanning, wideband NQR spectrometer for the VHF range. Journal of Magnetic Resonance, 286:148-157, 2018.

[3] R.K. Gupta, J.A. Ferretti, and E.D. Becker. Rapid Scan Fourier Transform NMR Spectroscopy. Journal of Magnetic Resonance, 13:275-290, 1974.

[4] Jeroen Elzerman and Mark Buitelaar. Quantum dots: Nuclear spins get a comb-over. Nature Physics, 12(7):634-635, 2016.

[5] A. M. Waeber, M. Hopkinson, I. Farrer, D. A. Ritchie, J. Nilsson, R. M. Stevenson, A. J. Bennett, A. J. Shields, G. Burkard, A. I. Tartakovskii, M. S. Skolnick, and E. A. Chekhovich. Few-second-long correlation times in a quantum dot nuclear spin bath probed by frequency-comb nuclear magnetic resonance spectroscopy. Nature Physics, 12(7):688-693, jul 2016.

[6] Djaudat Idiyatullin, Curt Corum, Steen Moeller, and Michael Garwood. Gapped pulses for frequency-swept MRI. Journal of Magnetic Resonance, 193(2):267-73, aug 2008. 
[7] M S Silver, R I Joseph, and D I Hoult. Highly Selective $\pi / 2$ and $\pi$ Pulse Generation. Journal of Magnetic Resonance, 59:347-351, 1984.

[8] M. S. Silver, R. I. Joseph, and D. I. Hoult. Selective spin inversion in nuclear magnetic resonance and coherent optics through an exact solution of the BlochRiccati equation. Physical Review A, 31(4):2753-2755, 1985.

[9] A. Abragam. Principles of nuclear magnetism. Oxford University Press, London, 1961.

[10] Djaudat Idiyatullin, Steven Suddarth, Curtis a Corum, Gregor Adriany, and Michael Garwood. Continuous SWIFT. Journal of Magnetic Resonance, 220:26-31, jul 2012.

[11] T. Kushida, G. B. Benedek, and N. Bloembergen. Dependence of the Pure Quadrupole Resonance Frequency on Pressure and Temperature. Physical Review, 104(5):1364-1377, dec 1956.

[12] H. W. De Wijn and J. L. De Wildt. Temperature dependence of nuclear quadrupole resonance in cuprous oxide. Physical Review, 150(1):200-201, oct 1966.

[13] K. Takeda. OPENCORE NMR: Open-source core modules for implementing an integrated FPGA-based NMR spectrometer. Journal of Magnetic Resonance, 192(2):218-229, jun 2008.

[14] R. K. Harris, E. D. Becker, S. M. Menezes, and D. E. Cabral. NMR nomenclature. Nuclear spin properties and conventions for chemical shifts(IUPAC Recommendations 2001). Pure and Applied Chemistry, 73(11):1795-1818, jan 2001. 